\title{
La era del big data en las relaciones públicas y su práctica profesional en Perú ${ }^{*}$
}

\author{
Luz María Flores Cabello** \\ Recibido: 2020-02-16 • Enviado a pares: 2020-03-10 \\ Aprobado por pares: 2020-04-21 • Aceptado: 2020-05-22 \\ https://doi.org/10.22395/angr.v19n37a8
}

\begin{abstract}
Resumen
El artículo pretende determinar cómo se encuentra la práctica profesional del relacionista público en Perú, en el contexto de la era del big data y la automatización digital, frente a los demás países de Latinoamérica. El objetivo de la investigación es conocer los beneficios y desventajas de la herramienta del big data en el ámbito de la comunicación estratégica y su uso en la práctica profesional. De igual manera, la finalidad de este artículo es conocer cómo está familiarizado el consultor peruano con estas herramientas para el logro de sus metas comunicacionales. Esta investigación aplicó un análisis de los resultados del trabajo desarrollado por Latin American Communication Monitor, el cual examina las tendencias y retos de la comunicación estratégica a través de un cuestionario aplicado a profesionales de relaciones públicas y gestión de comunicación. El resultado hallado en las estadísticas mostradas presenta que Perú, en contraste con otros países latinoamericanos, como Colombia y México, se encuentra en la cola de la automatización a causa de un reducido conocimiento del uso del big data en la elaboración de sus estrategias de comunicación. La información muestra que existe conciencia de su importancia, pero su uso es bajo entre los profesionales encuestados. Básicamente, este conocimiento se circunscribe al monitoreo de la analítica de datos para redes sociales y canales de comercio electrónico. La perspectiva que esta investigación abre a futuro es identificar la manera en que esta tecnología permite que la inteligencia se aplique en novedosas estrategias de comunicación corporativa.

Palabras clave: tecnología de la información; comunicación interactiva; relaciones públicas; comunicación móvil; comunicación de masas; grupos de interés; procesos de comunicación; información; comunicación.
\end{abstract}

\footnotetext{
Este artículo se desprende del Latin American Communication Monitor, organizado por Euprera, Dircom y Fundacom, que se enfoca en Perú durante los periodos bianuales 2016-2017 y 2018-2019. Estas encuestas a profesionales de la especialidad fueron aplicadas por la Facultad de Ciencias de la Comunicación de la Universidad de San Martín de Porres a través de las investigadoras doctoras Marita Solórzano y Patricia Bobadilla Terán. Esto se hizo a propósito de que la Escuela de Ciencias de la Comunicación es la única en Perú acreditada en la PRSA, además de que cuenta con un capítulo de estudiantes PRSSA, único en el país.

* Doctoranda en Periodismo, Universidad Complutense de Madrid, Madrid, España. Profesora e investigadora, Universidad de San Martín de Porres (USMP), Chiclayo, Perú. Correo electrónico: Ifloresc@usmp.pe. Orcid: https://orcid.org/0000-0003-4272-6419.
} 


\title{
The Time of Big Data in Public Relations and Its Professional Practice in Peru
}

\begin{abstract}
This article pretends to determine the current state of the professional practice of the public relations professional in Peru, in the context of the time of big data and digital automation in comparison with the other Latin-American countries. The goal of this research is to discover the benefits and disadvantages of the big data tool in the realm of strategic communication and its use in professional practice. Likewise, the main goal of this article is to get to know how familiarized the Peruvian professional is with these tools for the achievement of its communicational goals. This research analyzed the results of the work performed by Latin American Communication Monitor, who examines the trends and challenges in strategic communication through a questionnaire applied to public relations and communications management professionals. The statistical result displays that Peru, in contrast to other LatinAmerican countries such as Colombia and Mexico, is at the bottom in the use of automation due to a reduced knowledge in big data for the planning of communication strategies The information shows that there is an awareness of its importance, but its use is very limited among the polled professionals. This knowledge is circumscribed to monitoring the data analysis for social networks and e-commerce channels. The perspective opened by this research for the future is to identify how this technology allows intelligence to be applied to new corporate communication strategies.
\end{abstract}

Keywords: information technology; interactive communication; public relations; mobile communication; mass communication; groups of interest; communication processes; information; communication.

\section{A era do big data nas Relações Públicas e sua prática profissional no Peru}

\begin{abstract}
Resumo
Este artigo pretende determinar como a prática profissional do formado em Relações Públicas se encontra no Peru, no contexto do big data e da automatização digital, em comparação aos demais países latino-americanos. O objetivo da pesquisa é conhecer os benefícios e as desvantagens da ferramenta do big data no âmbito da comunicação estratégica e seu uso na prática profissional. Além disso, o artigo pretende conhecer como o consultor peruano está familiarizado com essas ferramentas para atingir suas metas comunicacionais. Esta pesquisa aplicou uma análise dos resultados do trabalho desenvolvido pelo Latin American Communication Monitor, o qual analisa as tendências e os desafios da comunicação estratégica por meio de um questionário aplicado a profissionais de Relações Públicas e Gestão da Comunicação. O resultado encontrado nas estatísticas apresenta que o Peru, em contraste com outros países da região, como a Colômbia e o México, encontra-se no final da lista da automatização por causa de um reduzido conhecimento do uso do big data na elaboração de estratégias de comunicação. A informação mostra que existe consciência de sua importância, mas seu uso é baixo entre os profissionais pesquisados. Esse conhecimento está relacionado com a monitoração da analítica dos dados para redes sociais e canais de comércio eletrônico. A perspectiva que a pesquisa abre para o futuro é como essa tecnologia permite que a inteligência seja aplicada em novas estratégias de comunicação corporativa.
\end{abstract}

Palavras-chave: tecnologia da informação; comunicação interativa; relações públicas; comunicação móvel; comunicação de massas; grupos de interesses; processos de comunicação; informação; comunicação. 


\section{Introducción}

La big data es una de las herramientas de la transformación digital de mayor impacto en el contexto tecnológico mundial en la actualidad. Esta nueva tecnología permite que las empresas y organizaciones conozcan, con un gran porcentaje de veracidad, variedad y en tiempo real, lo que les gusta y disgusta a sus clientes (McKinsey, 2011)

La transformación digital, basada en la nueva ciencia de datos, orilla a las empresas a adaptarse al poder que ejercen las nuevas tecnologías digitales para mantener la lealtad de sus clientes. Este nuevo panorama presenta desafíos en todos los ámbitos de la comunicación, lo que hace que la disciplina de las relaciones públicas se enfrente a una nueva manera de mirar. En este sentido, es necesario adaptarse a las formas innovadoras de comunicación estratégica que tienen las empresas hacia sus públicos (Wiencierz, 2019).

Los líderes corporativos tienen el rol de comunicar en dos vías dentro de sus organizaciones para construir la transparencia, los valores y la visión de la organización junto con sus colaboradores (Argenti, 2017, p. 156).

El objetivo de la presente investigación es conocer los beneficios y desventajas de la herramienta del big data en el ámbito de la comunicación estratégica y su uso en la práctica profesional. De igual manera, se intenta conocer si el consultor peruano está familiarizado con estas herramientas para el logro de sus metas comunicacionales.

Es de sumo interés para la especialidad del big data visibilizar la importancia de las tendencias tecnológicas en los nuevos canales de comunicación digital. Esto se puede hacer con base en estudios de investigadores de las relaciones públicas, como el Instituto de Relaciones Públicas (IPR), el McKinsey Institute, la consultora de tecnología Capgemini, entre otras. Estos estudios analizan el impacto del big data en la gran transformación digital del mundo de los negocios, el cual tiene un impacto específico en la profesión de las relaciones públicas. En su conjunto, estos análisis constituyen la fuente de información que ayuda a sustentar el marco teórico de la presente investigación.

Se han analizado las dos investigaciones bianuales realizadas por el Latin American Communication Monitor (LCM) en los años 2016-2017 y 2018-2019 (Moreno et al., 2017). Gracias a esta encuesta, realizada en diecisiete ${ }^{1}$ países en la primera edición y en diecinueve $^{2}$ en la segunda, y en la que participaron más de dos mil profesionales, se puede tener un acercamiento cualitativo del perfil competencial de los profesionales en los países latinoamericanos.

\footnotetext{
1 Argentina, Bolivia, Brasil, Chile, Colombia, Ecuador, Perú, Paraguay, Uruguay, Venezuela. Costa Rica, El Salvador, Guatemala, Honduras, Panamá, República Dominicana, México.

2 Argentina, Bolivia, Brasil, Chile, Colombia, Ecuador, Perú, Paraguay, Uruguay, Venezuela. Costa Rica, Cuba, El Salvador, Guatemala, Honduras, Nicaragua, Panamá, República Dominicana, México.
} 
En el caso de Perú resulta relevante encontrar un análisis de datos cuantitativos y de las tendencias profesionales de tal prestigio que, además, aporte a la investigación académica de la profesión. Es relevante si se considera que cuenta con una universidad reacreditada por la PRSA (Public Relations Society of America), lo cual incluye un capítulo de estudiantes de la PRSSA en su sede en Lima, Perú. La práctica y desarrollo de esta disciplina profesional, aún joven en la Latinoamérica, experimenta grandes cambios que es imprescindible investigar para el bien de la comunidad académica de las relaciones públicas.

\section{Definición de big data}

Se puede explicar y entender el concepto de big data si se tiene en cuenta la definición que lo describe como el conjunto de datos cuyo tamaño va más allá de la capacidad de procesamiento de un típico software. Los big data capturan, almacenan, manejan y analizan los datos, que pueden ser mayores a un terabyte (Arganza y Arroyo, 2018). La definición de su tamaño puede variar dependiendo de la clase de software que se utilice y del tamaño del conjunto de datos más común en una industria en particular, como exponía McKinsey Global Institute (2011) en el informe "Big data: The next frontier for innovation, competition and productivity". Para ese entonces, las compañías globales ya tenían la capacidad de capturar billones de bytes de información acerca de sus clientes, proveedores y procesos. Asimismo, podían tener millones de sensores incrustados en dispositivos, como los teléfonos móviles, automóviles o medidores inteligentes de energía (McKinsey, 2011, p. 5).

Con un enfoque diferente, Gartner Inc. - otra consultora de investigación en tecnologías de la información - definía el big data, en el año 2012, como un sistema de datos de gran volumen, alta velocidad o gran variedad de activos de información que demandan formas rentables e innovadoras de procesamiento de la información. Los big data permiten un mejor conocimiento, toma de decisiones y automatización de procesos (Pereira, Portilla y Rodríguez 2019).

Otro informe, "From big data to big insights. Blend innovation and governance to create an excellent customer experience", elaborado por Capgemini (2017), explica que el big data es un término que se ha disparado en los últimos años. En este informe también se afirma que algunos escépticos lo pueden ver como una reinvención de otros términos, como el análisis de flujo de datos. Sin embargo, big data es una cosa nueva por varios aspectos. En primer lugar, tiene acceso a muchos recursos de datos desde los dispositivos móviles, los sitios web, los metadatos y los sensores de objetos. En segundo lugar, los volúmenes de datos crecen exponencialmente. En tercer lugar, se puede hacer alguna cosa útil con estos datos. Y hay otras razones, porque la capacidad de las computadoras es enorme y barata como nunca antes (Capgemini, 
2017, p. 2). Capgemini, la consultora de servicios tecnológicos y transformación digital a nivel global, puntualiza que el valor de los proyectos de big data no siempre están a la altura, y las organizaciones aún no están explotando sus propias herramientas para conseguirlo. Esto quiere decir que la utilidad de la información obtenida de la tecnología del big data aún se encuentra en una etapa joven para las empresas.

De otro lado, la consultora norteamericana Boston Consulting Group estima que se produce un crecimiento de 2,5 EB de información al día. Aunque el término big data llega a ser confuso, no se refiere a un tamaño de información específica, ya que se utiliza cuando se habla en términos de petabytes (PB) y exabytes (EB). Estas unidades de medida equivalen a un trillón de bytes, es decir, es superior a un gigabyte o a un terabyte. Por ejemplo, un byte es una letra y diez bytes son dos palabras. Esto viene a ser la información diaria que se genera en el mundo de acuerdo con los censos poblacionales, datos de impuestos o registros médicos en los países (MonleonGetino, 2015, p. 429).

Según lo anterior, se puede decir que el big data es una herramienta que, aunque no analiza patrones, puede hacer probables predicciones de un acontecimiento (Weiner y Kochhar, 2016a). Por tanto, no solo se trata de hablar de una inmensa cantidad de datos, sino de comprender el concepto desde las características que lo constituyen. En este sentido, los expertos e investigadores del Institute for Public Relations analizan el concepto de big data a partir de cuatro características que lo constituyen, identificadas como las cuatro $v$ : volumen, velocidad, variedad y el valor. Por volumen se refiere a la cantidad de data que es generada y que puede variar de terabytes a cientos de petabytes. Por velocidad, la información generada en tiempo real y que es la que impulsa la transformación digital con mayor importancia para el éxito de la organización. En cuanto a la variedad, son los datos que pueden ser estructurados, no estructurados y semi estructurados y pueden afectar la forma cómo la organización los analiza. Y el valor es visto como la veracidad de la data que ayuda a reconocer patrones, derivar significados y hacer supuestos para la toma de decisiones, (Weiner y Kochhar, 2016a, p. 5).

En cuanto al volumen de los datos, hay que identificarlos por su naturaleza: estructurados, semiestructurados y no estructurados. Estos últimos son los que representan el gran reto para los expertos en la aplicación de técnicas que posibiliten su comprensión y análisis. Los datos estructurados pueden ser almacenados en tablas, como censos, encuestas, transacciones bancarias, compras en línea; están organizados de una forma mecanizada y manejable por simples algoritmos. Los datos semiestructurados son los que poseen una organización interna, como el software, las hojas de cálculo o los informes. Por contraste, los datos no estructurados carecen de formato determinado; son desordenados y no pueden ser almacenados en tablas, 
como los foros, las redes sociales, los correos electrónicos, las imágenes, el audio y el video, por lo que su obtención demanda alto costo (Weiner y Kochhar, 2016a, p. 10).

Tanto el big data como los algoritmos representan, entonces, una oportunidad de desarrollo de la comunicación estratégica, la cual se aleja de la comunicación tradicional para dar paso a un quehacer con funciones de mayor especialidad dentro de las organizaciones. De esta forma, la facilidad con la que las empresas u organizaciones logran conocer el comportamiento de sus públicos de interés es mucho mayor. Para el profesor Flores (2018), esta herramienta juega un papel fundamental en la búsqueda de datos e información digital para la investigación. En este sentido, afirma: "los algoritmos configuran cada vez más nuestro mundo, nuestro pensamiento, nuestra economía, nuestra vida política y nuestros cuerpos" (Flores, 2018, p. 275). En la figura 1 se detalla el funcionamiento del big data.

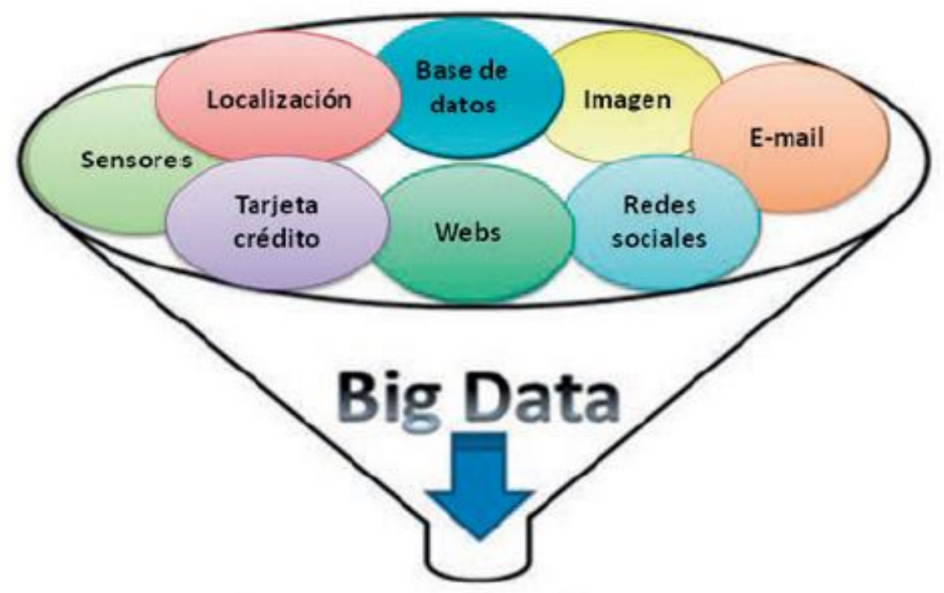

Algoritmos

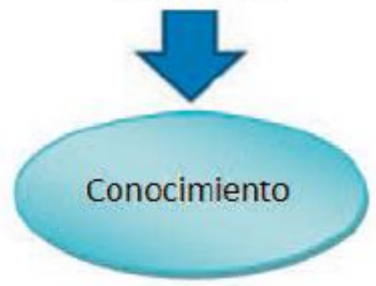

Figura 1. Funcionamiento del big data

Fuente: Gil (2016).

En este sentido, surgen voces sobre el nuevo contexto a las que es importante prestar atención. Este contexto está marcado por la presencia del poder del algoritmo en el quehacer de las relaciones públicas, tal como analiza Valentini (2019). Este autor 
señala que la tecnología está evolucionando a tal velocidad, que los académicos ya están muy por detrás de la práctica real de usar datos en las relaciones públicas. Esto quiere decir que se requiere poner atención, con relativa urgencia, a la evolución de la tecnología. La tecnología está lejos de ser un simple canal de comunicación. Es, más bien, un actor más inteligente que está cambiando la dinámica de la comunicación (Valentini, 2019).

\section{Retos del big data para las empresas}

Los avances de la tecnología de la automatización han desencadenado una serie de incertidumbres en torno al cuidado y la protección de la información privada y sensible de las personas. Este hecho pone en peligro la ética de muchas organizaciones públicas o privadas. Según el informe "El pasado, el presente y el futuro de los datos" de Dun y Bradstreet, publicado en el portal español It User (2019), una de cada cinco empresas asegura haber perdido clientes y oportunidades de crecimiento de ingresos debido a las malas prácticas de datos. El 20 \% reconoce que utilizó información incompleta o incompleta sobre ellos y un 15 \% dijo que no había firmado un nuevo contrato con un cliente por la misma razón. Por tanto, los tres mayores desafíos para que las empresas hagan uso de los big data son la protección de la privacidad de los datos, la obtención de datos precisos y el análisis o procesamiento de los datos. Sumado a ello, el 41 \% de los encuestados aseguró que "nadie en la organización es responsable de la gestión de los datos" (It User, 2019). El big data, como cualquier herramienta tecnológica, tiene beneficios para la sociedad de la información, pero también desventajas que afectan la lógica cuando se obvian razones éticas de seguridad y protección de la privacidad de las personas. Los beneficios más relevantes que ofrece el big data en la sociedad de la información son: i) capacidad de ofrecer una visión cada vez más precisa de las fluctuaciones y rendimientos de todo tipo de recursos, ii) permitir adaptaciones experimentales de un proceso a cualquier escala y conocer su impacto en tiempo real, iii) ayudar a conocer mejor la demanda con el fin de realizar una segmentación mucho más ajustada de la oferta para cada bien o servicio (Puyol, 2014).

No obstante, el análisis del big data presenta algunas limitaciones en su comprensión. Por ejemplo, algunos creen que estos pueden resolver los problemas del mundo. Pero lo que en realidad hace es buscar patrones recurrentes o correlacionar datos, lo cual no significa encontrar las causas. Esto quiere decir que los datos, por sí solos, no responden el porqué de las cosas que afirman (Weiner y Kochhar, 2016a).

En el libro Big data, privacidad y protección de datos, Gil (2016) explica algunos riesgos que vale la pena resaltar: i) caer en conclusiones erróneas que nadie revisa, ii) tomar decisiones automatizadas sin un sesgo humano, iii) vulnerar la privacidad de las personas, es decir, la protección de los datos personales. Monitorear los movimientos 
del comportamiento humano, a través de sus transacciones comerciales de todo tipo, en tiempo real y en cualquier parte del mundo, resulta una vulneración de la privacidad que los datos masivos podrían ocasionar para constituir una forma de control de poder sobre los seres humanos. Un caso real es el de China. En el 2020 se lanzará un programa para evaluar la confiabilidad de sus ciudadanos que dará un crédito social a partir de su comportamiento (Nuñez-Torrón, 2019).

Una mirada diferente a los retos que representa la privacidad de los datos masivos se encuentra en la postura del profesor Philipp Bachmann de la Universidad de Zúrich. Este autor se basa en los principios de la sociedad líquida del alemán Zygmunt Bauman para poner en duda la efectividad de la explosión de la tecnología digital en beneficio de las relaciones públicas, ya que genera indiferencia y ceguera moral. Este es el caso del escándalo de datos sucedido por Cambridge Analytica, el cual puede ser el prefacio de un futuro oscuro para las relaciones públicas digitales (Bachmann, 2019, p. 328).

\section{Usos del big data en las relaciones públicas}

Los usos del big data representan, en las relaciones públicas, una serie de aplicaciones para la profesión que permiten mejorar el análisis operativo interno y externo de la empresa. Con el uso del big data se expanden las oportunidades de remover las prácticas pasadas de comunicación, para crear mejores objetivos, mayor posicionamiento estratégico y una audiencia específica. Según Weiner y Kochhar (2016b, p. 2) el big data evoluciona las funciones de las relaciones públicas tradicionales permitiendo su crecimiento, eficiencia y eficacia, en aspectos tales como el análisis del entorno interno y externo, el planteamiento de los objetivos, el desarrollo de las estrategias, las tácticas y la evaluación. Es el valor que puede ser creado para mejorar la performance de la empresa, para una mejor comprensión de la competencia, los consumidores, los empleados, los medios y otros públicos. "Las organizaciones pueden aprender y reconocer que la data sola no responde el 'porqué' ni explica los insigths. Descubrir los insights de big data requiere el elemento humano y el pensamiento crítico para crear significado", (Weiner y Kochhar, 2016a, pág. 12).

Por su parte, Wiencierz (2019) resalta los aspectos más importantes en torno a las potencialidades del big data para las relaciones públicas. En este sentido, comienza por la forma en que pueden repercutir en la protección de la imagen de la marca, el manejo de la reputación de la empresa, la percepción de la marca por regiones demográficas, incluida la recepción de la competencia (p. 2). Además, se incluyen análisis de la cuota del mercado del cliente y el de la competencia, así como de su reputación. Una potencialidad, largo tiempo anhelada por las empresas, tiene que ver con la capacidad de examinar a gran detalle cómo, cuándo, dónde y por cuál punto el 
usuario busca la información acerca de la compañía o un tema en particular relacionado con esta. En este sentido, es necesario que el método analítico sea utilizado de acuerdo con la clasificación de los datos: estructurados o no estructurados; debe ser determinado por las metas a alcanzar en el primer momento de la investigación y no después. De acuerdo con esto, se destaca la importancia y relevancia de que el relacionista público no se limita a recolectar información para tener métricas de suficiencia, sino para establecer significados más profundos que los que ofrecen los datos: "[e]n otras palabras, no solo recolectamos información y sacamos métricas de vanidad, pero realmente tratamos de ir a la profundidad y establecer algunos significados que la información nos habla" (Wiencierz, p. 9).

En otro capítulo de esta investigación se precisa la importancia de hacer análisis para las relaciones públicas con base en metas claramente definidas, esto es, con preguntas que se relaciones directamente con el contenido. En caso de realizar el análisis situacional del share of voice, la cuota de participación del mercado requiere más un proceso diferente de generación de conocimiento de big data que un manejo de gestión de crisis. Hacer un análisis de big data en las relaciones públicas implica utilizar un método analítico que esté determinado por las metas de investigación de acuerdo con los patrones, las correlaciones o las tendencias (Wiencierz, 2019, p. 12). En este sentido, se debe analizar cómo se estructura este análisis y cuáles son sus valores típicos. El análisis descriptivo viene a ser el número de me gusta o visitas a la página web. Luego de este análisis, se recomienda analizar las razones de interrelación y los patrones que deben ser examinados en profundidad.

\section{Retos del aprendizaje del big data para los relacionistas públicos}

Todas las disciplinas del conocimiento están siendo impactadas por la transformación digital. La irrupción de la disrupción se impone como la nueva forma de resolver problemas y de adaptarse al cambiante mundo de las interrelaciones (Bustamante y Guillen, 2017). En este proceso, el reto del profesional de las relaciones públicas enfatiza en adaptarse a los nuevos conocimientos que configuran el manejo del big data, incluyendo sus características de volumen, velocidad, variedad y valor que la definen. El Latin American Communication Monitor 2016-2017 señala que el $45 \%$ de los profesionales encuestados en diecisiete países de la región reconocen que no tiene habilidades analíticas para manejar el big data. (Moreno, 2017).

En opinión Wiencierz (2017), muchos profesionales de la comunicación aún no son conscientes de la importancia de las tecnologías de la información, como es el uso del big data. Las potencialidades y los retos en el área de la comunicación corporativa aún están en debate, particularmente en términos de marketing y comunicación (p. 5). El panorama que transmiten es positivo, pero se reconoce que en el campo de las relaciones públicas hay un largo camino por recorrer. 
Bustamante y Guillen (2017) analizan las nuevas tendencias de perfiles profesionales que trae esta nueva ola de transformación tecnológica. De acuerdo con el informe del Randstad Professionals, hay un "perfil profesional altamente tecnológico, con experiencia internacional, polivalencia, adaptación al cambio, visión estratégica, capacidad analítica, grandes dotes de negociación y gestión y dominio del idioma inglés" (Bustamante y Guillen, 2017, p. 123). Esto quiere decir que la capacidad multidisciplinar que debe tener un profesional hoy en día, debe combinar los conocimientos informáticos, los tecnológicos y los comunicacionales.

Entre los nuevos perfiles de trabajo con tecnología big data destacan: el analista de datos, el estratega de datos, el científico de datos y el arquitecto de datos. Dos de ellos se acercan más a la disciplina de la comunicación:

- El analista de datos (data analyst) realiza funciones de consultas y reportes. Para ello, emplea herramientas analíticas, manejo de lenguajes de consultas y análisis estadísticos con los que obtiene métricas aplicadas al negocio. Sus conocimientos académicos se encuentran en la gama de las ciencias humanas como, por ejemplo, la sociología, las ciencias de la información, la investigación de mercados.

- El estratega de datos (data strategist) es un estratega corporativo; combina la estrategia global de la empresa con el conocimiento técnico.

En el análisis de las tendencias laborales en el campo profesional, se identifican los retos de la inteligencia artificial, los algoritmos, la comunicación visual, el management y la integración del mercadeo con la comunicación. Estos son los cambios interdisciplinarios más importantes en el futuro de la comunicación de las organizaciones (Rodríguez y Vásquez, 2019). Las oportunidades empresariales que se abren a partir del uso profesional de las redes sociales, así como la manera en que se han convertido en una herramienta para las estrategias de las empresas, evolucionan vertiginosamente. En este sentido, Elorriaga (2018) afirma: "Las nuevas tecnologías evolucionan muy rápido y pronto veremos cómo nuevas herramientas surgen. Las empresas y los nuevos perfiles laborales deberán adaptarse lo más rápido posible a las nuevas circunstancias".

\section{Metodología}

El presente artículo utiliza información relevante de las dos investigaciones bianuales realizadas por Latin American Communication Monitor (LCM) durante los periodos 2016-2017 y 2018-2019. Estas investigaciones tienen como objetivo analizar las prácticas actuales y el desarrollo futuro de la comunicación estratégica en diversos tipos de organizaciones y consultorías. El LCM es parte del proyecto Global Communication Monitor. Este es el mayor estudio longitudinal, en todo el mundo, sobre la gestión de comunicación y relaciones públicas que se realiza en Norteamérica, Asia-Pacífico y 
Latinoamérica. El LCM está organizado por la European Public Relations Education and Research Association (Euprera), con la Asociación de Directivos de Comunicación (Dircom) en el marco de Fundacom y con el mecenazgo de Llorente y Cuenca y la Universidad de Oregón (una en cada edición) (Moreno, 2017 y 2019). En ambos casos, la encuesta se realizó a traves de un cuestionario en línea que más de dos mil profesionales respondieron. Es importante precisar que los profesionales fueron invitados, en ambas ediciones, a través de correos electrónicos personales recogidos en las bases de datos de las principales asociaciones profesionales de cada país, así como de bases de datos propias del equipo de investigación.

La primera encuesta, "Tendencias en comunicación estratégica: big data, automatización, engagement, influencers, coaching y competencias", fue aplicada en diecisiete 3 países latinoamericanos a un total de dos mil doscientos noventa y cinco personas. Novecientos catorce profesionales completaron la encuesta en ejercicio efectivo de la profesión durante los meses de mayo y agosto de 2016. Se aplicaron treinta y tres preguntas agrupadas en quince secciones (Moreno, 2017, p. 10). Participaron un total de catorce universidades representadas por sus equipos de investigadores.

La segunda encuesta, "Comunicación estratégica y sus retos: fake news, confianza, información para la toma de decisiones, liderazgo, satisfacción, estrés y compromiso laboral", fue aplicada en diecinueve ${ }^{4}$ países latinoamericanos a un total de dos mil quinientos setenta y cinco. Mil ciento sesenta y cinco profesionales completaron la encuesta en ejercicio efectivo de la profesión durante los meses de abril y agosto de 2018. Se aplicaron treinta y nueve preguntas agrupadas en cinco secciones (Moreno, 2019, p. 10). Participaron un total de quince universidades representadas por sus equipos de investigadores que canalizaron el proceso.

En el caso específico de Perú, la investigación estuvo a cargo de la Universidad de San Martín de Porres. La Facultad de Ciencias de la Comunicación participó en ambas ediciones con el apoyo de las doctoras Marita Solorzano, en la primera edición, y Patricia Bobadilla en la segunda. En este punto cabe resaltar el rasgo característico de Global Communication Monitor, en cuanto a su "estricta selección de los encuestados, que lo diferencia de otros estudios de investigación que se basa en el muestreo por bola de nieve o que incluyen estudiantes, académicos, y personas de fuera de la profesión o región de estudio" (Moreno, 2017, p. 10).

El perfil profesional de los encuestados en general (no se distingue el país) en el periodo 2016-2017 es de 49,1 \% con más de diez años 26,2 \% de seis a diez años

\footnotetext{
3 Argentina, Bolivia, Brasil, Chile, Colombia, Ecuador, Perú, Paraguay, Uruguay, Venezuela. Costa Rica, El Salvador, Guatemala, Honduras, Panamá, República Dominicana, México.

4 Argentina, Bolivia, Brasil, Chile, Colombia, Ecuador, Perú, Paraguay, Uruguay, Venezuela. Costa Rica, Cuba, El Salvador, Guatemala, Honduras, Nicaragua, Panamá, República Dominicana, México.
} 
y el $24,7 \%$ con menos de cinco años de experiencia laboral. En cuanto al cargo, el $33,7 \%$ es director del departamento de comunicación corporativa u organizacional. El $26,3 \%$ es jefe de equipo o jefe de unidad. El 38,3\% es miembro del equipo consultor. En cuanto al género, el 66,6\% es femenino y el $33,4 \%$ es masculino; la media de edad fue de 39,8 años. El nivel de formación de magíster alcanzó el 53,2\%, mientras que el nivel de graduados llegó al 38,2 \%; tan solo 6 \% tiene el nivel de formación de doctor.

En el caso de la edición 2018-2019, el perfil profesional de los encuestados en general es de $22 \%$ con más de diez años, 22,1 \% de seis a diez años y el 55,9\% hasta cinco años de experiencia laboral. El cargo que ocupa el 36,5\% es director del departamento de comunicación corporativa u organizacional. El 28,2 \% se desempeña como jefe de equipo o jefe de unidad. El 35,3\% es miembro de equipo o consultor. En cuanto al género, un 60,1 \% es femenino y el 39,9\% es masculino; con una media de edad de 41,3 años. El nivel de formación de magister alcanza el 48,8 \%, mientras que de graduado llegó al 42,6 \%; tan solo 6,7 \% tiene el nivel de formación de doctor.

El objetivo de la presente investigación es conocer la herramienta del big data en el ámbito de la comunicación estratégica, su uso en la práctica profesional y si el consultor peruano está familiarizado con estas herramientas. Para ello, se realizó una consulta en septiembre 2019 a fin de confirmar si los temas planteados por el LCM seguían siendo vigentes o no un año después. En esta segunda etapa del estudio, se elaboró un cuestionario con tres preguntas con alternativas para marcar (tabla 1) que fue aplicado, con base en la información recogida de ambos LCM, a once profesionales de las relaciones públicas en ejercicio. La indagación permitió constatar si el uso del big data y la automatización en la gestión de la profesión son importantes entre los profesionales de relaciones públicas en Perú. De esta manera, se intentó confirmar si las tendencias seguirían vigentes en el 2021.

Del total de consultados, respondieron nueve personas (ocho ejercen su profesión en agencias de Lima y una ejerce en una agencia en Cusco). Cinco de sexo masculino y cuatro de sexo femenino, cinco con cargos de séniores y cuatro con cargos de consultores externos.

\section{Resultados}

Los temas analizados en ambos Latin American Communication Monitor (LCM) presentan una importante radiografía sobre los cambios tecnológicos que están impactando en la comunicación estratégica, así como la manera en que los países de la región se vienen adaptando a estos. Los profesionales encuestados en ambas ediciones coinciden en que el tema estratégico más importante en la gestión de la comunicación para los próximos tres años (hasta el 2021) será, en primer lugar, "enfrentarse con la evolución digital y la web social". 
Tabla 1. Cuestionario indagación del big data

\section{Tabla 1. Cuestionario indagación del big data}

\begin{abstract}
Pregunta
De los siguientes temas estratégicos ¿̇cuáles son los más importantes en la gestión de la comunicación de tus cuentas?
\end{abstract}

A. Enfrentarse con la evolución digital y la web social.

B. Usar big data o algoritmos para la comunicación.

C. Lidiar con la velocidad y volumen del flujo de información.

D. Fortalecer el rol de la función de la comunicación.

E. Conectar las estrategias de la organización y con la comunicación.

¿Has manejado alguna cuenta corporativa en la que ya se hayan implementado actividades de comunicación con el uso de big data en los últimos doce meses o más? Si la respuesta es afirmativa, señala en qué casos.

A. Revisión de la analítica de su canal de comercio electrónico.

B. Revisión de la analítica de sus redes sociales.

C.- Para adaptar contenidos.

D. Por favor detalla si no es ninguna de las anteriores.

¿Consideras útil para tu labor, el uso de algoritmos para desarrollar estrategias de comunicación más efectivas para tus clientes?

A. Para apoyar en la toma de decisiones de la gerencia.

B.- Para programar distribución de contenidos.

C. Para adaptar contenidos.

¿Si la respuesta es afirmativa, qué opciones usarían?

D. Por favor detalla si tu experiencia es otra.

Fuente: elaboración propia.

Los temas que siguen en segundo y tercer lugar en ambos informes presentan una variación. En el año 2018, la respuesta "lidiar con la velocidad y volumen del flujo de información" tenía un 36,2 \% de importancia, mientras que la respuesta "usar big data o algoritmos para la comunicación" alcanzó un 32,7 \%. Sin embargo, esta última respuesta ocupaba, dos años antes, el segundo lugar con un 37,8\% de importancia. Esto quiere decir que el interés descendió a un $5 \%$. Por otro lado, en el tercer lugar se ubicaba "fortalecer el rol de la función de comunicación" con 37 \%. En el cuarto lugar estuvo "lidiar con la velocidad y volumen del flujo de información" con un 32,6\% el cual toma un impulso y sube dos lugares dos años más tarde (ver figuras 2 y 2.1).

En ambos casos, los temas de mayor importancia tienen que ver con la gestión de la comunicación digital: "usar big data o algoritmos para la comunicación" y "lidiar con la velocidad y volumen del flujo de información".

Este flujo de temas a nivel regional cambia radicalmente cuando se enfoca en Perú, que tiene un orden de relevancia muy diferente. Por ejemplo, "conectar las estrategias de la organización con la comunicación" y "fortalecer el rol de la función 
de comunicación para apoyar la toma de decisiones de los altos directivos" son los más importantes para los encuestados peruanos, con 43,8 \% de interés. Le sigue la respuesta "uso del big data o algoritmos para la comunicación", con un 37,5\%. Sin embargo, para el siguiente periodo 2018-2019 este tema desciende en importancia hasta el $22 \%$, a pesar de que se ubica en el segundo puesto de temas estratégicos para la gestión de la comunicación. En el tercer lugar, se ubica el tema "enfrentarse con la revolución digital y la web social" con un $34,4 \%$. Este sube a $38 \%$ en el siguiente periodo y se ubica en el primer lugar de interés (ver tablas 2 y 2.1 ).

Enfrentarse con la evolución digital y la web social

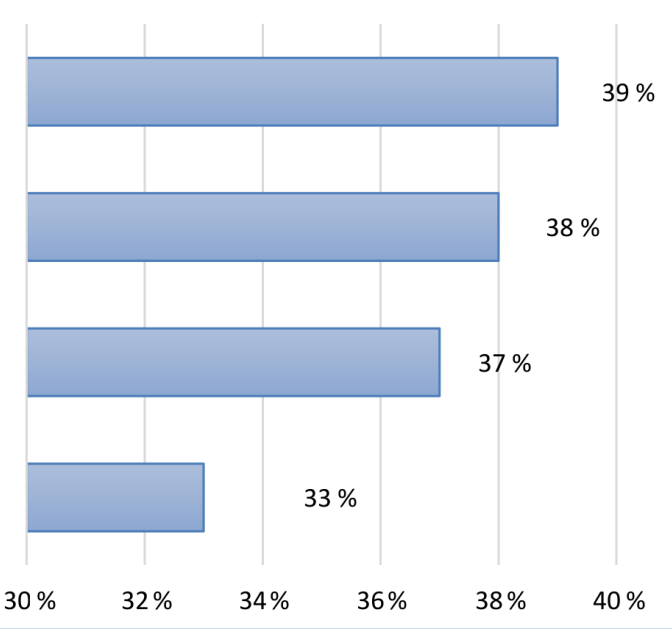

Fortalecer el rol de la función de la comunicación

Lidiar con la velocidad y volumen del flujo de información

Figura 2. Temas estratégicos más importantes en la gestión de la comunicación hasta 2021 Fuente: Moreno (2019, p. 46).

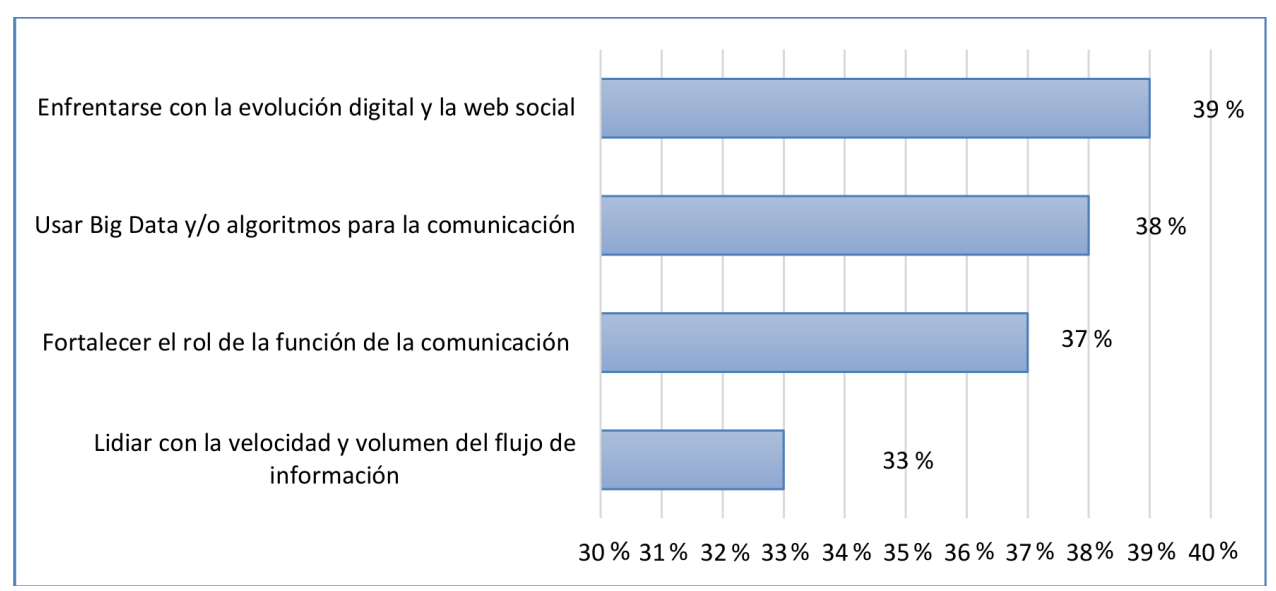

Figura 2.1. Temas estratégicos más importantes en la gestión de la comunicación hasta 2019

Fuente: Moreno (2017, p. 83). 
Tabla 2. Relevancia percibida de los temas estratégicos en Latinoamérica

\begin{tabular}{|c|c|c|c|c|c|}
\hline & $\begin{array}{l}\text { Conectar las } \\
\text { estrategias de la } \\
\text { organización con } \\
\text { la comunicación }\end{array}$ & $\begin{array}{l}\text { Fortalecer el rol } \\
\text { de la función de } \\
\text { comunicación } \\
\text { para apoyar la } \\
\text { toma de decisiones } \\
\text { de los altos } \\
\text { directivos }\end{array}$ & $\begin{array}{l}\text { Uso del big data o } \\
\text { algoritmos para la } \\
\text { comunicación }\end{array}$ & $\begin{array}{l}\text { Enfrentarse con la } \\
\text { revolución digital } \\
\text { y la web social }\end{array}$ & $\begin{array}{c}\text { Lidiar con la } \\
\text { velocidad y el } \\
\text { volumen del flujo } \\
\text { de información }\end{array}$ \\
\hline México & $34,4 \%$ & $34,4 \%$ & $35,4 \%$ & $47,9 \%$ & $37,5 \%$ \\
\hline Costa Rica & $38,0 \%$ & $36,0 \%$ & $36,0 \%$ & $54,0 \%$ & $26,0 \%$ \\
\hline R. Dominicana & $31,7 \%$ & $25,4 \%$ & $42,9 \%$ & $50,8 \%$ & $20,6 \%$ \\
\hline Argentina & $30,9 \%$ & $30,0 \%$ & $40,9 \%$ & $33,6 \%$ & $40,0 \%$ \\
\hline Brasil & $29,3 \%$ & $28,7 \%$ & $32,8 \%$ & $30,5 \%$ & $43,7 \%$ \\
\hline Chile & $40,0 \%$ & $38,9 \%$ & $26,3 \%$ & $35,8 \%$ & $26,3 \%$ \\
\hline Colombia & $23,9 \%$ & $50,0 \%$ & $40,1 \%$ & $37,3 \%$ & $29,6 \%$ \\
\hline Perú & $43,8 \%$ & $43,8 \%$ & $37,5 \%$ & $34,4 \%$ & $28,1 \%$ \\
\hline Venezuela & $28,3 \%$ & $37,7 \%$ & $58,5 \%$ & $41,5 \%$ & $35,8 \%$ \\
\hline
\end{tabular}

Fuente: Moreno (2017, p. 84).

En Perú los temas estratégicos varían en importancia entre el primer y segundo LCM.

Tabla 2.1. Importancia percibida de los temas estratégicos en distintos países de Latinoamérica

\begin{tabular}{|c|c|c|c|c|c|}
\hline & $\begin{array}{c}\text { Construir y mantener } \\
\text { la confianza en la } \\
\text { organización }\end{array}$ & $\begin{array}{c}\text { Conectar las } \\
\text { estrategias de la } \\
\text { organización con la } \\
\text { comunicación }\end{array}$ & $\begin{array}{l}\text { Enfrentarse con la } \\
\text { evolución digital y } \\
\text { la web social }\end{array}$ & $\begin{array}{l}\text { Usar big data o } \\
\text { algoritmos para la } \\
\text { comunicación }\end{array}$ & $\begin{array}{l}\text { Lidiar con la } \\
\text { velocidad y } \\
\text { volumen del flujo } \\
\text { de información }\end{array}$ \\
\hline Perú & $38 \%$ & $18 \%$ & $38 \%$ & $22 \%$ & $32 \%$ \\
\hline
\end{tabular}

Fuente: Moreno (2019, pp. 49-50).

\section{Tendencias de comunicación estratégica}

En adelante, el análisis se centra en el LCM 2016-2017, cuyo foco de estudio se basa principalmente en las tendencias de la comunicación estratégica como el big data y la automatización. Inicialmente los temas de importancia estratégica son liderados por la opción "enfrentarse con la evolución digital y la web social", seguido por el "uso del big data y algoritmos en las comunicaciones". En este sentido, es necesario conocer cómo los profesionales miran las actividades de big data en el quehacer laboral. Por ejemplo, es notoria la variación de indicadores, con respecto al primer ítem —referido a la adaptación de algoritmos de servicios en línea-, de Perú frente a México. 
Este ítem ocupa el primer lugar de importancia para los encuestados de Perú, con 71 \% frente a los casi 79 \% para México. Sin embargo, ambos países lo usan solo en un $38 \%$ y 38,5\% respectivamente, mientras que en Colombia la relación entre importancia y uso son más cercanas (ver tabla 3).

Tabla 3. Percepción sobre la importancia de algoritmos en la comunicación estratégica en las regiones latinoamericanas

\begin{tabular}{|c|c|c|c|c|c|c|c|c|c|c|}
\hline & \multicolumn{2}{|c|}{$\begin{array}{c}\text { Adaptación de } \\
\text { algoritmos de servicios } \\
\text { en línea como motores } \\
\text { de búsqueda }\end{array}$} & \multicolumn{2}{|c|}{$\begin{array}{l}\text { Herramientas } \\
\text { algorítmicas } \\
\text { programadas para } \\
\text { apoyar la toma de } \\
\text { decisiones }\end{array}$} & \multicolumn{2}{|c|}{$\begin{array}{l}\text { Herramientas } \\
\text { algorítmicas } \\
\text { programadas para } \\
\text { la distribución de } \\
\text { contenido }\end{array}$} & \multicolumn{2}{|c|}{$\begin{array}{l}\text { Herramientas } \\
\text { algorítmicas } \\
\text { programadas para } \\
\text { la adaptación de } \\
\text { contenido }\end{array}$} & \multicolumn{2}{|c|}{$\begin{array}{c}\text { Herramientas } \\
\text { algorítmicas } \\
\text { programadas para la } \\
\text { creación de contenido }\end{array}$} \\
\hline & $\begin{array}{c}\text { Importan- } \\
\text { cia }\end{array}$ & Uso & $\begin{array}{c}\text { Importan- } \\
\text { cia } * *\end{array}$ & Uso* & $\begin{array}{c}\text { Importan- } \\
\text { cia }\end{array}$ & Uso & $\begin{array}{c}\text { Importan- } \\
\text { cia }^{* *}\end{array}$ & Uso & $\begin{array}{c}\text { Importan- } \\
\text { cia }\end{array}$ & Uso \\
\hline México & $78,6 \%$ & $38,5 \%$ & $66,7 \%$ & $30,4 \%$ & $74,4 \%$ & $38,8 \%$ & $64,0 \%$ & $22,4 \%$ & $62,8 \%$ & $34,3 \%$ \\
\hline Colombia & $65,9 \%$ & $45,2 \%$ & $55,9 \%$ & $20,5 \%$ & $68,5 \%$ & $52,3 \%$ & $45,5 \%$ & $25,5 \%$ & $58,3 \%$ & $39,3 \%$ \\
\hline Perú & $71,2 \%$ & $38,0 \%$ & $61,0 \%$ & $30,0 \%$ & $62,1 \%$ & $60,8 \%$ & $49,2 \%$ & $24,0 \%$ & $63,8 \%$ & $41,2 \%$ \\
\hline
\end{tabular}

Fuente: Moreno (2017, p. 39).

En la segunda etapa del estudio realizado entre profesionales de Perú en septiembre 2019, se verificó que lo más importante para los relacionistas públicos consultados es conectar las estrategias de la organización con la comunicación. Ante la pregunta de si habían realizado alguna "implementación de acciones de big data en la gestión de las relaciones públicas en los últimos doce meses", cuatro de los consultados señalaron que no han implementado acciones de big data porque no lo manejan o no lo usan. Por otro lado, quienes han implementado estas acciones explican que lo han hecho a través de la revisión analítica de sus redes sociales y de la analítica de su canal de comercio electrónico.

Ante la consulta sobre la importancia del "uso de algoritmos para desarrollar estrategias de comunicación más efectivas en su quehacer diario", todos admitieron que es importante.

\section{Discusión y conclusiones}

Frente al reto de la transformación digital y del conocimiento de los beneficios y desventajas que tiene el big data en el ámbito de la comunicación estratégica, el análisis realizado deja entrever varios aspectos importantes. En primer lugar, el relacionista público de Perú tiene un amplio trecho por avanzar, ya que se ubica en el último puesto de la automatización entre los países latinoamericanos. En segundo lugar, se confirma que los profesionales en relaciones públicas tienen algunas debilidades 
sobre el conocimiento de la herramienta del big data, sus beneficios y utilidades para el ejercicio de la profesión. Finalmente, las prioridades en el ejercicio de su profesión no pasan prioritariamente por "enfrentarse a la evolución digital y la web social", según la proyección al 2021. Más bien, su prioridad es "conectar las estrategias de la organización con la comunicación", es decir, hay una mirada más general con respecto a los demás países latinoamericanos que priorizan su labor ante la evolución digital. No obstante, cabe aclarar que los relacionistas de Perú tampoco son ajenos al avance de la tecnología digital, pero esta se circunscribe básicamente al ámbito de la analítica para redes sociales y los canales de comercio electrónico. Sin embargo, esto no significa con ello que se trabaje con estrategias en comunicación corporativa basadas en la inteligencia del big data.

Por otro lado, en cuanto al estudio y análisis del LCM, se confirma que la definición de este término no es reconocida por todos. Antes bien, más del $50 \%$ de los encuestados del LCM 2017 tiene una definición incorrecta del término big data. Es cierto que existe entre los consultados del LCM un conocimiento más informado sobre el uso de esta tecnología. Sin embargo, hay un punto de inflexión cuando se aprecia que muchas empresas aún no implementan esta herramienta en su desarrollo ni saben cuál es el rol de un relacionista público en ello. Tal como se registra en la investigación de los LCM, más de la mitad de los encuestados admiten que no han planeado el inicio de actividades de big data antes que termine 2017 ni se han implementado ya estas actividades.

\section{Desafíos profesionales}

En un repaso a los desafíos de los profesionales para trabajar con big data, casi la mitad reconoce su falta de habilidades técnicas para manejarlos. Asimismo, se carece de tiempo suficiente para analizar los datos y de un software que encaje con las necesidades de comunicación. Quedan entre los últimos lugares las preocupaciones éticas, que apenas fueron tomadas en cuenta por algún grupo encuestado.

Por último, cabe preguntarse si hace falta una mayor conciencia de las empresas y organizaciones para entender la transformación digital a la que se orienta el mundo. Además, teniendo en cuenta que el componente ético es poco visible para los encuestados, las empresas serían las únicas que pueden garantizar certidumbre de confianza en esta nueva era de automatización de la sociedad. En conclusión, Weiner y Kochhar (2016b) lo explican de esta manera: "Para los profesionales de relaciones públicas, los vientos de cambio están soplando. Quienes se adapten tendrán éxito, mientras que quienes ignoren la importancia emergente de la tecnología, la ciencia y las relaciones públicas de datos, lamentablemente desaparecerán." (p. 3, traducción propia). 


\section{Referencias}

Arganza, R. y Arroyo, M. (2018). ¿Qué es big data? Marketing XXI. https://www.marketing-xxi.com/ big-data-aplicaciones-gestion-dato-distintas-etapas-funnel-conversion/big-data

Argenti, P. (2017). Strategic communication in the C-Suite. International Journal of Business Communication, 54(2), 146-160.

Bachmann, P. (2019). Public relations in liquid modernity: How big data and automation cause moral blindness. Public Relations Inquiry, 8(3), 319-331. https://doi.org/10.1177/2046147X19863833

Bustamante, N. y Guillen, S. (2017). Un acercamiento al big data y su utilización en comunicación. Mediaciones Sociales, 16, 115-134. https://doi.org/10.5209/MESO.58112

Capgemini, I. (2017). From big data to big insights. Blend innovation and agile governance to create an excellent customer experience. Estados Unidos.

Elorriaga, A. (2018). El social big data: una oportunidad empresarial y laboral. Estudios sobre el Mensaje Periodístico, 24(2), 1213-1222. https://doi.org/10.5209/ESMP.62210

Flores, J. (2018). Algoritmos, aplicaciones y big data, nuevos paradigmas en el proceso de comunicación y de enseñanza-aprendizaje del periodismo de datos. Revista de Comunicación, 17(2), 268-291. https://doi.org/10.26441/RC17.2-2018-A12

Gil, E. (2016). Big data, privacidad y protección de datos. Agencia Española de Protección de Datos.

IT User. (2019, 10 de julio). Los tres mayores retos de big data para las empresas. IT User. https:// discoverthenew.ituser.es/predictive-analytics/2019/07/los-3-mayores-retos-de-big-data-paralas-empresas

McKinsey Global Instituto. (2011). Big Data: The next frontier for innovation, competition and productivity. McKinsey \& Company.

Monleon-Getino, A. (2015). El impacto del big data en la sociedad de la información. Significado y utilidad. Historia y Comunicación Social, 20(2), 427-445. https://doi.org/10.5209/rev_HICS.2015. v20.n2.51392

Moreno, A. M. (2017). Latin American Communication Monitor 2016-2017. Tendencias en comunicacion estratégica: big data, automatización, engagement, influencers, coaching y competencias. Resultados de una encuesta en 17 países. Euprera/Dircom.

Moreno, A. M. (2019). Latin American Communication Monitor 2018-2019 Comunicacion Estratégica y sus retos: fake news, confianza, información para la toma de decisiones, liderazgo y compromiso laboral. Resultados de una encuesta en 19 países. Euprera/Dircom.

Nuñez-Torrón, A. (2019, 27 de julio). El sistema de crédito social en China: una distopía hecha realidad. Tic Beat. https://www.ticbeat.com/empresa-b2b/el-sistema-de-credito-social-en-china-unadistopia-hecha-realidad/

Pereira, T., Portilla, I., y Rodríguez, N. (2019). Big data y Relaciones Públicas. Una revisión bibliográficadel estado de la cuestión. Revista de Comunicación, 18(1), 151-165. https://doi. org/10.26441/RC18.1-2019-A8

Puyol, J. (2014). Una aproximación al big data. Revista de Dereho UNED, (14), 471-505. 
Rodríguez, L. y Vázquez, P. (2019). Retos y perspectivas en la comunicación organizacional. El profesional de la Información, 28(5), 1-7. https://doi.org/10.3145/epi.2019.sep.01

Valentini, C. (2019). Theories in Public Relations: Reflections and Future Directions. Public Relations Inquiry, 8(3), 195-200. https://doi.org/10.1177/2046147X19881227

Weiner, M. (2016). Irreversible: The public relations big data revolution. Public Relations Society of America: https://instituteforpr.org/irreversible-public-relations-big-data-revolution/

Weiner, M. y Kochhar, S. (2016a). Irreversible: The public relations big data revolution. Public Relations Society of America: https://instituteforpr.org/irreversible-public-relations-big-data-revolution/

Weiner, M., y Kochhar, S. (2016b). On the Horizon: Unleashing the Power of Big Data in Public Relations. Obtenido de Public Relations Society of America: https://apps.prsa.org/Intelligence/ TheStrategist/Articles/view/1 1678/1152/On_the_Horizon_Unleashing_the_Power_of_Big_ Data_in\#.XleJ6XlKgdW

Wiencierz, C. (2017). The use of big data in corporate communication. Corporate Communications: An internal journal, 22(3), 258-272. 10.1108/CCIJ-02-2016-0015

Wiencierz, C. (2019). Big Data in Public Relations: a Conceptual framework. Public Relations Journal, 12(3), 15. 